\title{
RAPID SMOKING, CUE EXPOSURE AND SUPPORT IN THE MODIFICATION OF SMOKING
}

\author{
Martin Raw and M. A. H. Russell \\ Addiction Research Unit, Institute of Psychiatry, 101 Denmark Hill, London SE5 8AF, England
}

(Received 18 December 1979)

\begin{abstract}
Summary-Forty-nine dependent smokers were assigned to one of three treatment conditions: rapid smoking, cue exposure or simple support. There were no differences between treatments in the proportion who stopped smoking or the average reduction in smoking at the end of treatment or at $1 \mathrm{yr}$ follow-up. The overall proportion who stopped was $22 \%$ at the end of treatment and $14 \%$ at $1 \mathrm{yr}$ follow-up. Time of the first cigarette of the day (before tea or coffee) and percentage reduction in consumption whilst trying to cut down unaided, predicted abstinence at $1 \mathrm{yr}$ follow-up. These results do not support claims made for the effectiveness of rapid smoking.
\end{abstract}

For many years researchers have sought an effective way to help people stop smoking. Various facts have emerged. Firstly, although end of treatment success (abstinence) rates can be high, with claims as high as $100 \%, 1 \mathrm{yr}$ after treatment they are dramatically lower with most studies around $20 \%$ (Raw, 1978). Most people who stop smoking during treatment relapse during the first 3 months following treatment (Hunt and Matarazzo, 1973). Another fact concerns the issue of specific vs nonspecific treatment effects. A specific treatment effect can be defined in the following way. A specific treatment $(A)$ is compared with simple support (B) and a no-treatment control (C). A specific treatment effect exists only when $A$ is better than B and C. If A and B are equally effective but better than $\mathrm{C}$ a nonspecific treatment effect has been demonstrated. Although treatment is more effective than no-treatment (Bernstein, 1970; Keutzer, 1968; Russell, Armstrong and Patel, 1976), no specific treatment has been shown to be more effective than simple support or attention placebo (Raw, 1978). This suggests that when people do stop smoking during treatment is is because of the nonspecific factors which apply to all treatments, including simple support. McFall and Hammen (1971) have described some of these factors as motivation, self-monitoring and the structure and support offered by treatment. If this is true, the precise technique used in treatment does not matter provided the other so-called 'nonspecific' ingredients are present.

Rapid smoking has been described as an "outstanding exception" to the generally disappointing trend of low success rates and high relapse rates (Danaher, 1977). Early studies by Lichtenstein and his co-workers obtained 6 month follow-up success rates of $57 \%$ and $53 \%$ respectively* (Schmahl, Lichtenstein and Harris, 1972; Lichtenstein et al., 1973). However, they acknowledged in the 1972 paper that "the data on treatment components are not clearly interpretable" and in a more recent review of the evidence Lichtenstein and Danaher (1976) conceded: "It must be acknowledged that the magnitude of the rapid smoking effect does not appear to be large". Indeed the 1973 study compared rapid smoking with two other conditions derived from the earlier study, namely warm smoky air (blown into the face of the smoker contingent upon smoking), and warm smoky air plus rapid smoking, and found all three to be equally effective. Another study (Harris and Lichtenstein, 1971) investigated the relative importance of rapid smoking by giving all subjects the same rapid smoking procedure but varying the amount of social support they received in addition. Subjects received high social support (verbal reinforcement of abstinence, warmth/friendliness, positive expectations) or low social support. At 3 months follow-up $72 \%$ of the 'high' support group were abstinent

* These success rates are based on the assumption that dropouts (from whom no follow-up data were obtained) were failures. 
compared with $6 \%$ of the 'low' support group, suggesting that rapid smoking, per se, added little to the nonspecific components of the treatment. In spite of this evidence Lichtenstein and Danaher (1976) persist in claiming that "rapid smoking per se seems to be a significant variable". Several other studies, however, have failed to show rapid smoking to be better than other methods or controls, and most studies by other workers have achieved considerably lower success rates. Lando (1975) for instance found that rapid smoking was no more effective than satiation or an attention-placebo control at $1 \mathrm{yr}$ follow-up, with an overall abstinence rate of $36 \%$ at the end of treatment and $20 \%$ at 1 yr follow-up. Sutherland et al. (1975), Glasgow (1977) and Saterfield (1977) obtained 6 month follow-up success rates for rapid smoking far below those obtained by Lichtenstein and co-workers at $0 \%, 7 \%$ and $10 \%$, respectively. These studies did not publish abstinence rates at $1 \mathrm{yr}$ follow-up. The evidence in favour of a clinically relevant specific effect of rapid smoking therefore seems to be weak. We have investigated the issue in this study by comparing rapid smoking with simple support and a new procedure, cue exposure, which we developed in the hope that it would prevent relapse.

Cue exposure is based on the assumption that certain events (cues or stimuli) have become associated with smoking and can act as stimuli for it. Examples of such events are cups of tea or coffee, other people offering cigarettes, and the sight and smell of the cigarette itself. Cue exposure involves exposing smokers to the cues which make them want to smoke and preventing the response. This should produce extinction of the response although other processes such as alteration of expectations and learning of self-control are undoubtedly involved (Hodgson and Rankin, 1976). Indeed cue exposure sounds a sensible treatment on purely practical grounds, since it involves the smoker in facing and coping with the sorts of cues he or she will have to face in real life. This is why it should help prevent relapse, since most of the cues described above will be present after treatment, tempting the smoker to smoke again.

Finally, although the main purpose of this study was to assess the effectiveness of rapid smoking and cue exposure, we were also interested to see whether certain subject variables would predict outcome. We set out then to answer the following questions:

(1) Are rapid smoking and cue exposure more effective than simple support?

(2) Does cue exposure prevent relapse?

(3) What subject variables, if any, predict outcome?

\section{METHODS}

\section{Subject selection}

Subjects were 49 people drawn from the Maudsley Hospital Smokers Clinic, who smoked 34.9 cigarettes per day and were $39.5 \mathrm{yr}$ old on average. Twenty were men and 29 women. About half were referred to the clinic by a doctor and about half were selfreferred, usually having heard about the clinic from a friend. After their first contact with the clinic they were sent two questionnaires. When these were returned they were offered a $1 \mathrm{~h}$ assessment interview at which their health, smoking history and motivation to stop smoking were discussed. They then took home a record sheet on which they were asked to record their normal smoking over $24 \mathrm{~h}$ of a typical day. It was emphasized that they should continue smoking in their usual way and make no attempt to cut down at this stage. When they returned this record they were sent a booklet in which to record their smoking for a week, during which they were to try to cut down and if possible stop smoking. Both records were returned by post. The five people who stopped at the 1 week booklet stage were excluded from the trial. Only when people had completed all these stages of assessment were they put on the waiting list for treatment. The average time on the waiting list was 3 months.

Table 1 shows how the 49 people who started treatment were selected from 151 cases seen at the initial assessment interview. Almost half the sample failed to complete the two 
Table 1. 151 people from whom 49 were treated

\begin{tabular}{lr}
\hline Seen at initial interview & 151 \\
Already abstinent at this interview & 3 \\
Failed to return 24 hr smoking record & 47 \\
Failed to return 1 week booklet & 26 \\
Stopped during assessment & 5 \\
Declined participation in trial & 10 \\
Accepted treatment but stopped before first session & 6 \\
Accepted treatment but failed to attend first session & 5 \\
Treated in trial & 49 \\
\hline
\end{tabular}

self recording procedures. Altogether $88(58 \%)$ dropped out and $14(9 \%)$ stopped smoking before treatment, leaving $49(32 \%)$ who started treatment.

Of 10 people who declined to participate in the trial four did so because they did not want group treatment and six because they lived too far away to attend regularly. The specific treatment approach was not discussed at the initial assessment interview. We explained that they would be seen in small groups and that the details of treatment would be explained at the first treatment session. The letter offering treatment gave no details of the treatment procedure other than the days, dates and times of the sessions, but it did ask them to bring a $f 10$ (\$25) cash deposit which was to be returned provided they attended all sessions: $£ 5$ at the end of treatment and $£ 5$ at 6 months follow-up.

\section{Assignment to groups}

As soon as at least 15 people had completed assessment and were on the waiting list, they were assigned to one of three treatments. Four cohorts of 15 people were offered treatment. These were taken in over a 13 month period. The dropout rate between being offered treatment and the first treatment session was evenly balanced between the treatments, being four for support (two stopped), three for cue exposure (two stopped) and four for rapid smoking (two stopped). Assignment to the treatments was balanced for sex and consumption. One additional constraint was placed on allocation because of the potential risks of rapid smoking for people with coronary heart disease (Hauser, 1974; Russell et al., 1978). Men over 40 and women over 50 were allocated only to support or cue exposure. Of the treated subjects, 16 received support, 17 cue exposure and 16 rapid smoking.

\section{The treatment schedule}

The attendance schedule was designed with most sessions in the first week and the time between sessions growing progressively longer as treatment proceeded. This is shown in the diagram.

\begin{tabular}{lccccccc}
\hline Week & & 1 & & 2 & 3 & 4 \\
Treatment session & 1 & 2 & 3 & 4 & 5 & 6 & 7 \\
Day & Wed Thur & Fri & Mon & Fri & Wed & Wed \\
\hline
\end{tabular}

The treatment course lasted 3 weeks. Each subject came for treatment at the same time each day. The time of each treatment was varied across the four cohorts to ensure that time of day was not confounded with treatment. The treatment groups consisted of 4-5 subjects each. Each group session lasted $45 \mathrm{~min}$ and the same therapist saw all groups. At the beginning of the first session the details and rationale of treatment were explained and the cash deposit collected. Subjects were encouraged to stop smoking completely from that moment on.

\section{Data collection}

Baseline consumption was reported on the initial questionnaires. Subjects recorded their daily consumption on cards from the beginning of the first week (i.e. 2 days before 
the first treatment session) for 6 weeks. They were followed up by post after 3 months, attended a follow-up session at the clinic after 6 months and were contacted again by post after $1 \mathrm{yr}$. At the beginning of the first treatment session a venous blood sample was taken to determine blood nicotine and carboxyhaemoglobin ( $\mathrm{COHb})$ levels. Samples were also taken, without prior warning, at the penultimate treatment session (6th) and the 6-month follow-up session as a check on claimed abstinence. For the rapid smoking group an extra sample was taken at the end of the first session to determine the rise in $\mathrm{COHb}$ and nicotine levels after rapid smoking. These data are reported elsewhere (Russell et al., 1978). Mood data were collected at the beginning and end of each treatment session using a shortened scale derived from McNair and Lorr (1964). The two highest loading items were chosen from each of their scales except Aggression (Anger) from which three items were chosen. The scales were Anxiety, Aggression, Activity, Friendliness, Fatigue, Self-esteem and Ability to Concentrate. 'Desire for a smoke' was also rated on a scale from 0 (none) to 6 (very strong). Both the mood and desire measures were collected three times each treatment session: firstly to describe mood/desire "on average over the last three days" or "since the last treatment session" (whichever was shorter). This was done at the beginning of the session. Then to describe mood/desire 'now' at the beginning of the session, and the third time to describe mood/desire 'now' at the end of the session.

\section{Details of the three treatments}

Support. This included record keeping, encouragement and support from the therapist including verbal praise for abstinence, reassurance about the difficulties of withdrawal and advice on how to cope with them, mutual support and encouragement of fellow group members. The advice was directive and subjects were told to avoid cues whenever possible (for instance to avoid the smoking compartments of buses and trains).

Cue exposure. In addition to the basic ingredients described above, subjects received practice in cue exposure. Throughout the 3 weeks of treatment they kept their cigarettes and were encouraged to confront cues (like people smoking in the smoking compartments of buses and trains) whenever possible. Within sessions they were three or four times instructed to light up cigarettes, sometimes taking a non-inhaled puff, then leave the cigarette burning, sometimes in their hand, sometimes in the ashtray. Other cues associated with smoking, like cups of tea and coffee, were also introduced. The therapist sometimes offered cigarettes (to give subjects practice at refusing) and sometimes lit cigarettes himself. Finally, on each day following a treatment day they were instructed to practice cue exposure in a favourite smoking situation three times; once in the morning, afternoon and evening. Immediately before practising cue exposure they filled in the mood scale. As a control for this extra task the support and rapid smoking groups filled in the mood scale on the same three occasions (i.e. in their favourite smoking situations) the day following a treatment day, but did not perform any other task.

Rapid smoking. We followed Lichtenstein's rapid smoking procedurc as closely as possible by using his instructions guide (Lichtenstein, 1975). Subjects were instructed to light a cigarette and inhale on command every $6 \mathrm{~s}$. They continued this until they could tolerate no more, even if it meant smoking more than one cigarette. When they reached their tolerance limit they said "Stop" and put out the cigarette. They then had a $5 \mathrm{~min}$ break before repeating the procedure, and if they could tolerate it had a further break and further repetition. In practice very few subjects needed more than one repetition. There was very little talk during the rapid smoking but time for supportive talk after it since most subjects could only tolerate about $20-25$ min of the procedure.

\section{RESULTS}

Because men over 40 and women over 50 were excluded from rapid smoking there was an age difference in the three treatment groups $(45.2,39.9$ and $33.4 \mathrm{yr}$ for support, cue exposure and rapid smoking respectively, $p<0.01$ ). We therefore tested for a relationship between age and outcome to determine whether all subjects could be included in the 
Table 2. Basic consumption variables: means

\begin{tabular}{lcccc}
\hline & \multicolumn{4}{c}{ Treatment groups } \\
& All Ss & Support & Cue exposure & Rapid smoking \\
\hline Cigarette consumption & & & & \\
Baseline & 34.9 & 34.9 & 37.1 & 32.6 \\
24 h recorded & 29.0 & 29.4 & 28.8 & 28.6 \\
1 week reduced & 22.2 & 25.1 & 19.5 & 21.6 \\
Beginning-of-treatment & 19.6 & 23.1 & 18.4 & 16.9 \\
End-of-treatment & 13.1 & 14.8 & 9.5 & 14.6 \\
3 month follow-up & 23.2 & 25.1 & 20.5 & 23.8 \\
6 month follow-up & 25.0 & 23.8 & 23.1 & 28.3 \\
1 yr follow-up & 24.8 & 25.1 & 25.3 & 23.9 \\
Blood measures & & & & 8.1 \\
Pre-treatment COHb (\%) & 8.3 & 8.5 & 8.4 & 4.7 \\
End-of-treatment COHb (\%) & 4.5 & 4.3 & 4.5 & 29.2 \\
Pre-treatment nicotine (ng/ml) & 34.6 & 37.7 & 35.6 & \\
\hline
\end{tabular}

Consumption is the average number of cigarettes smoked per day. Beginning-of-treatment consumption was the level recorded on the first treatment day, and end-of-treatment consumption was the daily mean during the fourth treatment week. The consumption data are based on the 44 subjects for whom end of treatment data were available $(16,14$ and 14 respectively): the mising cases dropped out of treatment. Pre- and end-oftreatment $\mathrm{COHb}$ is based on 40 subjects who provided complete data $(13,15$ and 12 respectively) and nicotine on 43 cases $(15,16$ and 12 respectively). None of the between group differences in the table was significant at the $5 \%$ level.

analysis. There was no relation between age and outcome (average reduction in consumption or proportion of people who stopped smoking) either within treatments or for all subjects pooled.

\section{Changes in mean consumption}

Table 2 shows the consumption data before, during and after treatment. Average consumption for the total sample fell from 35 cigarettes per day (cpd) to $13 \mathrm{cpd}$ at the end of treatment (a $63 \%$ reduction) and $25 \mathrm{cpd}$ at 1 yr follow-up ( $29 \%$ reduction). The three treatments achieved a reduction of $58 \%$ (Support), $74 \%$ (Cue exposure) and $55 \%$ (Rapid smoking) at the end of treatment. Neither end-of-treatment consumption nor percentage reduction in consumption showed significant differences between treatments $\left(\chi^{2}=1.87\right.$ and 3.20 respectively; Kruskal-Wallis one-way analysis of variance). This was also true of percentage reduction in consumption at 3 months, 6 months and $1 \mathrm{yr}$ follow-up $\left(\chi^{2}=2.56,2.56\right.$ and 0.25 , respectively).

\section{Number of people abstinent in each treatment group}

Table 3 shows the percentage and number of people abstinent in each treatment group. Altogether $22 \%$ were abstinent at the end of treatment, falling to $14 \%$ at $1 \mathrm{yr}$ follow-up (people who dropped out of treatment were counted as failures). There were no significant differences between treatments in the number of people abstinent at the end of treatment or at 3 month, 6 month and $1 \mathrm{yr}$ follow-up $\left(\chi^{2}=1.49,1.17,1.17\right.$ and 1.26 respectively, $2 d f$ in each case).

Table 3. Success rates of the three treatment groups shown as the percentage of people who stopped smoking completely (actual numbers in parenthesis).

\begin{tabular}{lcccc}
\hline & All Ss & \multicolumn{2}{c}{ Treatment groups } \\
& $N=49$ & $\begin{array}{c}\text { Support } \\
N=16\end{array}$ & $\begin{array}{c}\text { Cue exposure } \\
N=17\end{array}$ & $\begin{array}{c}\text { Rapid smoking } \\
N=16\end{array}$ \\
\hline End of treatment & $22(11)$ & $19(3)$ & $29(5)$ & $19(3)$ \\
3 months follow-up & $14(7)$ & $19(3)$ & $18(3)$ & $6(1)$ \\
6 months follow-up & $14(7)$ & $19(3)$ & $18(3)$ & $6(1)$ \\
1 yr follow-up & $14(7)$ & $19(3)$ & $18(3)$ & $6(1)$ \\
\hline
\end{tabular}




\section{Carboxyhaemoglobin levels}

For the 40 subjects who provided end of treatment data mean $\mathrm{COHb}$ fell from $8.3 \%$ to $4.5 \%$ and there were no significant differences between treatments (Table 2).

\section{Overall consumption changes}

Although there were no significant differences between treatments in consumption, there was an overall treatment effect (see Table 2 and Fig. 1). The consumption changes can be broken down into four stages.

(1) A self-monitoring effect. When recording their normal smoking subjects reported a mean daily consumption of 29 , compared with 35 reported on the initial questionnaire. This reduction of $17 \%$ was significant $(t=4.81, d f 43, p<0.001)$.

(2) A self-control effect. When subjects were asked to cut down their smoking as much as possible while keeping a record of their attempt in the 1 week booklet, they reported a mean daily consumption of 22 . This further reduction of $24 \%$ was also significant $(t=4.34, d f 43, p<0.001)$. At this stage the overall reduction from baseline was $36 \%$.

(3) $A$ treatment effect. From the beginning to the end of treatment subjects reduced from 20 to $13 \mathrm{cpd}$, a reduction of $35 \%$ (Wilcoxon, $p<0.001$ ). End of treatment consumption was $63 \%$ lower than baseline.

(4) Relapse. Consumption increased after the end of treatment to $23 \mathrm{cpd}$ at 3 months follow-up $(t=5.77, d f 43, p<0.001)$. The further increase to 25 at 6 month and $1 \mathrm{yr}$ follow-up was not significantly different from the 3 month level. The mean consumption of $25 \mathrm{cpd}$ at 1 yr follow-up was significantly lower than baseline consumption $(\mathrm{t}=4.96$, df $43, \mathrm{p}<0.001$ ) but not lower than the $24 \mathrm{~h}$ recorded consumption of $29 \mathrm{cpd}$. The reduction at $1 \mathrm{yr}$ follow-up was not due to treatment successes alone (end of treatment consumption $=0 \mathrm{cpd}$ ) since when these were excluded the treatment failures still smoked less at 1 yr follow-up than at baseline (29 cpd compared with $36 \mathrm{cpd} ; t=3.78$, df 33 , $p<0.01$ ). This is shown in Fig. 1. In. view of this we further divided treatment 'failures' into those who reduced their smoking at the end of treatment by less than $75 \%(N=25)$ and those who reduced by $75 \%$ or more $(N=9)$ to see if a 'clinically significant' end of treatment reduction was maintained. Those who cut down during treatment by $75 \%$ or more maintained a significant reduction at $1 \mathrm{yr}$ follow-up (23 cpd compared with a baseline of $37 \mathrm{cpd} ; t=4.4$, df $12, p<0.001$ ). However, those who cut down by less than $75 \%$ during treatment did not maintain a significant reduction (31 cpd compared with a baseline of $35 \mathrm{cpd}$ ).

Figure 1 shows that the treatment effect appeared very early. Treatment successes were smoking less than failures at the 1 week booklet stage before treatment (Mann-Whitney, $p<0.05)$, and on the first $(p=0.02)$ and second $(p<0.001)$ treatment days. Virtually all the reduction in consumption achieved by the end of treatment was achieved by the

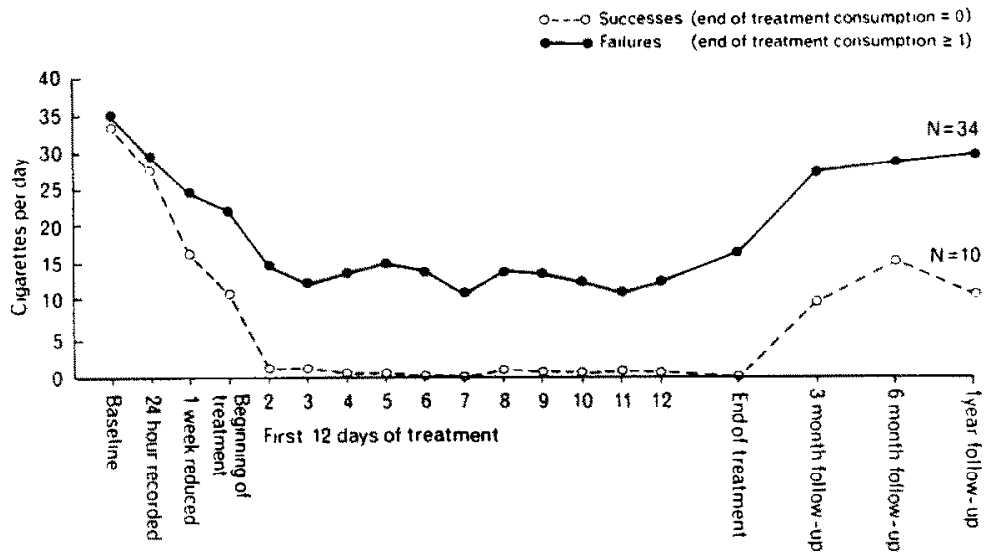

Fig. 1. Average daily cigarette consumption during assessment, treatment and follow-up of treatment 'successes' and 'failures'. Note: the difference between successes and failures is significant at the 1 week reduced stage (Mann-Whitney, $p=0.04$ ) and thereafter. 


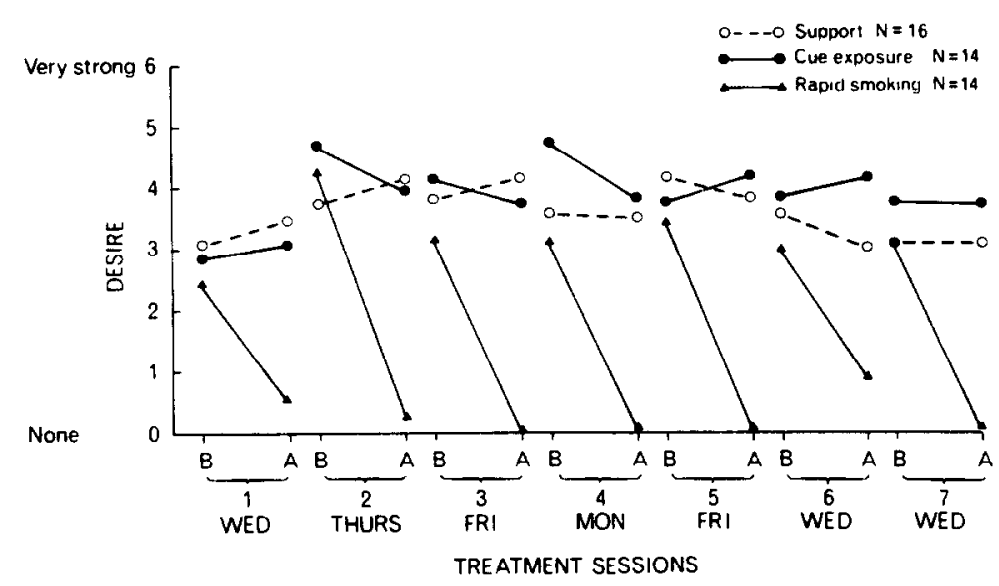

Fig. 2. 'Desire for smoke-now' at the beginning and end of treatment sessions for the three treatments.

second day of treatment, and smoking status on the second day strongly predicted smoking status at one year follow-up $\left(\chi^{2}=9.68,1 d f, p<0.01\right)$, as did cigarette consumption $(r=0.55, p=0.001)$.

\section{Mood and 'desire for a smoke' data}

There were no significant differences between treatments or changes during treatment in any of the seven mood factors. Nor did any of the mood factors change during treatment for treatment successes. The 'desire for a smoke-now' measure clearly showed the effect of rapid smoking (Fig. 2). Whilst it sometimes increased and sometimes decreased during treatment sessions for support and cue exposure, it consistently and markedly went down for the rapid smoking group to virtually 'none'. However at the beginning of each treatment session after the first, 'desire for a smoke-now' in the rapid smoking group had returned to its previous level, indicating the relatively short lived subjective effect of rapid smoking.

The ratings collected at the beginning of each treatment session to describe "desire for a smoke--over the last 3 days or since the last treatment session" were compared in treatment successes and failures. Since virtually all treatment successes stopped smoking immediately (Fig. 1) their ratings reflect desire to smoke in abstainers. Neither treatment successes nor failures showed a significant reduction in these ratings of desire to smoke during the 3 weeks of treatment. Throughout treatment the failures had a stronger desire to smoke than the successes: 4.9 compared with 4.2 at the beginning of treatment (MannWhitney, $p<0.05)$ and 4.5 compared with 3.1 at the end of treatment $(\mathrm{M}-\mathrm{W}, p<0.005)$.

\section{Prediction of outcome}

All four pre-treatment consumption variables (see Table 2) were correlated with consumption at the end of treatment and at $1 \mathrm{yr}$ follow-up, and with each other (the lowest $r$ was $0.44, p<0.01$ ). Four other pre-treatment variables which we considered might reflect strength of 'dependence' were also related to short or long term outcome. One was an item on the initial questionnaires which asked "In the morning do you smoke your first cigarette before tea or coffee-YES/NO". Of the 29 who answered YES only one was abstinent at $1 \mathrm{yr}$ follow-up whilst of the 20 who answered NO, 6 were (Fisher's exact test, $p<0.05$ ). I his item was correlated with the pre-treatment consumption measures. Two others were ratings given at the beginning of the first treatment session. "Desire to smoke, on average over the last 3 days" scored from 0 (none) to 6 (very strong), and "How confident are you that you will succeed in giving up smoking - not at all, only slightly, moderately, fairly strongly, very strongly?". The desire rating was correlated with consumption at the end of treatment $(r=29, p<0.05)$ but not at 1 yr follow-up $(r=0.08)$ and did not correlate with any of the pre-treatment consumption variables. Confidence was not cor- 
related with end of treatment consumption $(r=-0.16)$ but was related to consumption at $1 \mathrm{yr}$ follow-up $(r=0.35, p<0.05)$. The other variable was pre-treatment carboxyhaemoglobin $(\mathrm{COHb})$ which was correlated with consumption at the end of treatment $(r=0.40, p<0.01)$ and at 1 yr follow-up $(r=0.33, p<0.05)$. Sex was not related to smoking status at short or long term follow up.

Since the consumption variables were highly inter-correlated. baseline consumption was selected to represent them. Baseline consumption, percentage reduction in consumption whilst trying to cut down (1 week reduced consumption in Table 2 ) and the other four pre-treatment variables described above were included in a multiple regression analysis with smoking status (abstinent or not) at $1 \mathrm{yr}$ follow-up as the dependent variable. Only percentage reduction in consumption whilst trying to cut down and time of the first cigarette (before tea or coffee) significantly predicted long term outcome (multiple $R=0.56, F(2,41)=9.2, p<0.001)$, accounting for $31 \%$ of the variance. Baseline consumption, pre-treatment $\mathrm{COHb}$, desire to smoke and confidence in ability to stop only added another $4 \%$ to the variance accounted for.

\section{DISCUSSION}

Our results clearly show that neither rapid smoking nor cue exposure was more effective than simple support; and cue exposure did not prevent relapse. We suggested in the introduction that Lichtenstein's own work, especially the Harris and Lichtenstein (1971) study, did not support his claim for the effectiveness of rapid smoking. Indeed, it suggested the relative unimportance of rapid smoking compared with the nonspecific factors of motivation, structure, support and encouragement. This study supports that conclusion. In terms of percentage reduction in smoking or abstinence rates, at the end of treatment and at follow-up, there were no differences between rapid smoking, cue exposure and simple support. This was in spite of our evidence (Fig. 2) that rapid smoking eliminated the desire to smoke immediately after a treatment session.

Although our overall success rate was lower than those reported in the early studies of Lichtenstein and co-workers, it is consistent with those reported in the other four studies mentioned in the introduction. In his review of rapid smoking research, Danaher (1977) suggests that different success rates with rapid smoking may be due to procedural differences. Whilst we used only one therapist for all treatments, and saw people in small groups (4 or 5), the two more successsful Lichtenstein studies (Schmahl et al., 1972; Lichtenstein et al., 1973) saw people individually and used several therapists. However, Glasgow (1978), whose overall success rate at 6 months follow-up was $16 \%$, also used several therapists and treated people individually. It is possible that the warning Lichtenstein et al.'s subjects received, that if they smoked between sessions they would be excluded from treatment (Schmahl, personal communication), might account for the different outcome between our study and theirs. Obviously, such an instruction could increase motivation to stop smoking or to claim abstinence. Since in their studies "reports of abstinence received praise, self-disclosure and encouragement while reports of continued smoking were met with contingent admonishment" (Danaher, 1977) there may have been considerable pressure on subjects to lie, and the only check on claims of abstinence was the report of an informant. In contrast, we checked reports of abstinence by measuring carboxyhaemoglobin levels and as a result reclassified one man, who claimed to have switched from cigarettes to non-inhaled pipe smoking, as a failure because his $\mathrm{COHb}$ level was $6 \%$. However, there was no outright lying by our treatment successes (one case was borderline ${ }^{*}$ ) and obviously the importance of such validation

\footnotetext{
- In a study by Cole (1975), the mean COHb level of 100 office workers who were smokers in central London was $5.5 \%$ with a range of $2.2-13.0 \%$. In 100 non-smoking office workers the mean COHb level was $1.1 \%$ with a range of $0.1-2.7 \%$. All but a few of the non-smokers had COHb levels of $2.0 \%$ or less. In our study all but two of our treatment successes were below 2.0\%: one had a level of 2.0 and another of $2.5 \%$. Both were counted as successes but $2.5 \%$ lies in the borderline between smoking and non-smoking which probably requires further checking. Russell, Cole and Brown (1973) showed that after $78 \mathrm{~min}$ exposure to cigarette smoke in an unventilated room, the mean $\mathrm{COHb}$ level of 12 non-smokers was $2.6 \%$ with a range of $1.7 \%$ to $4.2 \%$. Thus it is possible for non-smokers to have a 'smoking' level under certain circumstances.
} 
depends on how strongly the treatment setting tempts people to lie by making them feel guilty about failing. Such lying can be common (Ohlin, Lundh and Westling, 1976).

A more likely source of differences between studies is differences in the subject population treated, although again the data are equivocal. Whilst our smokers averaged 40 yrs of

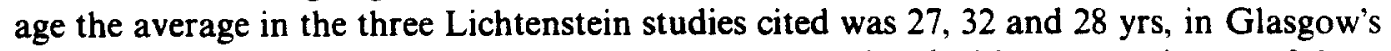
study 33 yrs and in Lando's 31 yrs, but age was not correlated with outcome in any of these studies. Cigarette consumption seems a possible factor, expecially as it correlated strongly with outcome in our study. Our subjects were very heavy smokers, smoking nearly twice the national average for smokers in Britain (18.4 for men and $14.4 \mathrm{cpd}$ for women; HMSO 1976). Average consumption in the three Lichtenstein studies was 29, 27 and $26 \mathrm{cpd}$, in Glasgow's study $31 \mathrm{cpd}$ and in Lando's study $32 \mathrm{cpd}$, although both Lichtenstein et al. and Glasgow report no correlation between consumption and outcome (Lando does not report any findings on the prediction of outcome). Whether or not consumption is a good index of dependence, our smokers were certainly extremely dependent since they were only treated if they failed to stop unaided before treatment, and only one third of our initial interviewees was actually treated (see Table 1). By contrast most of the other cited studies recruited subjects through posters and newspaper adverts and started treatment very quickly.

That degree of dependence might explain differences between studies is suggested by our prediction of outcome results. The two variables which predicted long term outcome (smoking status at $1 \mathrm{yr}$ follow-up) represented consumption and the ability to cut down unaided. Time of first cigarette was correlated with baseline consumption, and in fact, when cigarette consumption at $1 \mathrm{yr}$ follow-up was used as the dependent variable in the multiple regression instead of smoking status, the only differences in the results were that multiple $R$ went up a little and baseline consumption took the place of time of first cigarette as a predictor. If it is not surprising that consumption should predict consumption at follow-up, or that the ability to cut down unaided should predict smoking status at follow-up, it is surprising that the other studies cited did not find these correlations. One possible explanation is that these factors predicted outcome because the success rate was so low, i.e. a powerful treatment was not masking individual differences. Presumably this is also why the treatment effect appeared so early in our study, and the successes were already smoking less than the failures at the 1 week booklet stage before treatment. It meant that in our study long term outcome was determined very early indeed, by the second day of treatment. These results certainly make Danaher's (1977) suggestion that "any participant has an equal chance of success" seem unlikely.

Acknowledgements-We would like to thank Jean Crutch for secretarial help, the Department of Health and Social Security and the Medical Research Council for financial support, and Colin Taylor for statistical advice. We also thank the following, for their helpful advice and comments: Dick Eiser, Ray Hodgson. Martin Jarvis. Howard Rankin and Stephen Sutton.

\section{REFERENCES}

BERNSTEIN D. A. (1970) The modification of smoking behaviour: a search for effective variables. Behat. Res. Ther. 8, 133-146.

COLE P. V. (1975) Comparative effects of atmospheric pollution and cigarette smoking on carboxyhaemoglobin levels in man. Nature, London 255, 699-701.

DANAHER B. G. (1977) Research on rapid smoking: interim summary and recommendations. Addictite Behavs 2, 151-166.

GLASGOW R. E. (1979) The effects of a self-control manual and amount of therapist contact in the modification of smoking behaviour. Unpublished Ph.D., University of Oregon.

HARRIS D. E. and LICHTENSTEIN E. (1971) The contribution of nonspecific social variables to a successful behavioral treatment of smoking. Paper read to Western Psychological Association. USA.

HAUSER R. (1974) Rapid smoking as a technique of behaviour modification. Caution in selection of subjects. $J$. consult. clin. Psychol. 42, 625-626.

HMSO (1976) The General Household Survey.

HoDGSON R. and RANKIN H. J. (1976) Modification of excessive drinking by cue exposure. Behat. Res. Ther. 14, 305-307.

Hunt W. A. and Matarazzo J. D. (1973) Recent developments in the experimental modification of smoking behaviour. J. abnorm. Psychol. 81, 107-114.

KEUTZER C. S. (1968) Behaviour modification of smoking: the experimental investigation of diverse techniques. Behav. Res. Ther. 6, 137-157. 
LANDO H. A. (1975) A comparison of excessive and rapid smoking in the modification of chronic smoking behaviour. J. consult. clin. Psychol. 43, 350-355.

LICHTENSTEIN E. (1975) Rapid smoking: a procedures guide. Unpublished guide.

LiCHTENSTEIN E. and DANAHER B. G. (1976) Modification of smoking behaviour: a critical analysis of theory, research and practice. In Progress in Behaviour Modification Vol. 3. (Edited by HeRSEN M., EISLER R. M. and Miller P. M.). Academic Press, New York.

Lichtenstein E., Harris D. E., Birchler G. R., Wahl J. M. and Schmahl D. P. (1973) Comparison of rapid smoking, warm smoky air, and attention placebo in the modification of smoking behaviour. $J$. consult. clin. Psychol. 40, 92-98.

MCFALL R. M. and HAMmen C. L. (1971) Motivation, structure and self-monitoring: role of non-specific factors in smoking reduction. J. consult. clin. Psychol. 37, 80-86.

MCNAIR D. M. and LORR M. (1964) An analysis of mood in neurotics. J. abnorm. Psychol. 69, 620-627.

OHLIN P., LUNDH B. and WestuING H. (1976) Carbon monoxide blood levels and reported cessation of smoking. Psychopharmacology 49, 263-265.

Raw M., Israel Y., Glaser F. B., Kalant H., Popham R. E., Schmidt W. and Smart R. G. (1978) The treatment of cigarette dependence. In Research Advances in Alcohol and Drug Problems, Vol. 4. (Edited by IsRael, Y. ). Plenum, New York.

Russell M. A. H., Armstrong E. and Patel, U. A. (1976) Temporal contiguity in electric aversion therapy for cigarette smoking. Behav. Res. Ther. 14, 103-123.

Russell M. A. H., Cole P. V. and Brown E. (1973) Absorption by non-smokers of carbon monoxide from room air polluted by tobacco smoke. Lancet $00,576-579$.

Russell M. A. H., Raw M., TAylor C., Feyerabend C. and Saloojee Y. (1978) Blood nicotine and carboxyhaemoglobin levels after rapid smoking aversion therapy. J. consult. clin. Psychol. 46, 1423-1431.

SATERFIELD H. D. (1977) Efficacy of self-instruction as a treatment component in smoking cessation. Unpublished Ph.D., Stanford University.

SCHMAHL D. P., LichtensteIN E. and HaRRis D. E. (1972) Successful treatment of habitual smokers with warm smoky air and rapid smoking. J. consult. clin. Psychol. 36, 105-111.

Sutherland A., Amit Z., Golden M. and Roseberger Z. (1975) Comparison of three behavioural techniques in the modification of smoking behaviour. J. consult. clin. Psychol. 43, 443-447. 